\title{
On Hadamard Matrices Constructible by Circulant Submatrices
}

\author{
By C. H. Yang
}

\begin{abstract}
Let $V_{2 n}$ be an $H$-matrix of order $2 n$ constructible by using circulant $n \times n$ submatrices. A recursive method has been found to construct $V_{4 n}$ by using circulant $2 n \times 2 n$ submatrices which are derived from $n \times n$ submatrices of a given $V_{2 n}$. A similar method can be applied to a given $W_{4 n}$, an $H$-matrix of Williamson type with odd $n$, to construct $W_{8 n}$. All $V_{2 n}$ constructible by the standard type, for $1 \leqq n \leqq 16$, and some $V_{2 n}$, for $n \geqq 20$, are listed and classified by this method.
\end{abstract}

Let $H_{n}$ be an $n \times n$ Hadamard matrix. Although it is conjectured that no circulant $H_{4 n}$-matrix exists for $n>1$ (see [3]), it is known that many $H_{4 n}$-matrices can be constructed by using circulant submatrices of order $n$ or $2 n$. (For $H$-matrices of Williamson type, see [1], [2], [4].)

Let $V_{2 n}$ be an $H_{2 n}$-matrix constructible by using circulant $n \times n$ submatrices. Then $V_{2 n}$ can be constructed by the following standard type:

$$
M_{2 n}=\left[\begin{array}{cc}
A & B \\
-B^{T} & A^{T}
\end{array}\right], \quad \text { where } A, B \text { are } n \times n \text { circulant matrices }
$$

and $C^{T}$ means the transposed matrix of $C$.

A recursive method has been found to construct $V_{4 n}$ by circulant $2 n \times 2 n$ matrices which are derived by circulant $n \times n$ submatrices of a given $V_{2 n}$. (See Theorem 1 , below.) Likewise, let $W_{4 n}$ be an $H_{4 n}$-matrix of Williamson type with odd $n ; W_{8 n}$ can be constructed by using $2 n \times 2 n$ symmetric circulant matrices which are derived from $n \times n$ symmetric circulant submatrices of a given $W_{4 n}$. (See Theorem 2.)

Let $S_{n}=\left(\left(e_{i}\right)\right)$ be the $n \times n$ circulant matrix with the first row entries $e_{i}$, $(0 \leqq$ $i \leqq n-1)$, all zero except for $e_{1}=1$. Then $n \times n$ circulant matrices $A, B$ of (*) can be written as polynomials in $S$. (We shall omit the suffix $n$ of $S_{n}$ and others when there is no confusion.)

$$
A=A_{n}(S)=\sum_{i=0}^{n-1} a_{i} S^{i}, \quad B=B_{n}(S)=\sum_{i=0}^{n-1} b_{i} S^{i},
$$

with coefficients $a_{i}, b_{i}=1$ or -1 ; where $S^{0}=I_{n}=$ the $n \times n$ identity matrix.

A sufficient condition for the matrix $M_{2 n}$ of type (*) being an $H$-matrix is that $M_{2 n} M_{2 n}^{T}=2 n I_{2 n}$ which is equivalent to

$$
A A^{T}+B B^{T}=2 n I_{n} .
$$

Received March 31, 1970.

AMS 1970 subject classifications. Primary 05B20, 62K05, 05A19; Secondary 15A36, 0504, 1504.

Key words and phrases. Construction of Hadamard matrices, circulant matrices, standard type $\boldsymbol{H}$-matrices, Williamson type $\boldsymbol{H}$-matrices, recursive method for $\boldsymbol{H}$-matrices, table for some $\boldsymbol{H}$-matrices. 
Let $P=P_{n}(S), Q=Q_{n}(S)$ be matrices obtained by replacing -1 by 0 in $A, B$ respectively. Then the condition (1) is equivalent to

$$
P P^{T}+Q Q^{T}=\left(p_{n}+q_{n}-r_{n}\right) I+r_{n} J,
$$

where $J=J_{n}=\sum_{i=0}^{n-1} S^{i}$ and $p_{n}, q_{n}$ are, respectively, the numbers of 1's in each row of $P, Q$. Here, $p_{n}, q_{n}$ and $r_{n}$ must be solutions of the following necessary conditions for existence of $V_{2 n}$.

$$
\begin{gathered}
\left(n-2 p_{n}\right)^{2}+\left(n-2 q_{n}\right)^{2}=2 n, \\
p_{n}+q_{n}-r_{n}=\frac{1}{2} n .
\end{gathered}
$$

Similarly, by taking $Q^{\prime}=J-Q$, instead of $Q$ in (2), (3), and (4), which is possible since whenever $A$ and $B$ satisfy the condition (1), so do $A$ and $-B$, we obtain the corresponding conditions:

$$
\begin{gathered}
P P^{T}+Q^{\prime} Q^{\prime T}=\left(p_{n}+q_{n}^{\prime}-r_{n}^{\prime}\right) I+r_{n}^{\prime} J, \\
\left(n-2 p_{n}\right)^{2}+\left(n-2 q_{n}^{\prime}\right)^{2}=2 n, \\
p_{n}+q_{n}^{\prime}-r_{n}^{\prime}=\frac{1}{2} n .
\end{gathered}
$$

Since $q_{n}^{\prime}=n-q_{n}$, we also obtain from (7) and (4),

$$
r_{n}^{\prime}=2 p_{n}-r_{n}
$$

THEOREM 1. Let $M_{2 m}$ be a given $V_{2 m}$-matrix of type $\left(^{*}\right)$ satisfying the conditions (2), (3), and (4). Then $M_{4 m}, a V_{4 m}$-matrix of type (*), can be found as follows:

$$
P_{2 m}(s)=P_{m}\left(s^{2}\right)+s^{k} Q_{m}\left(s^{2}\right), \quad Q_{2 m}(s)=P_{m}\left(s^{2}\right)+s^{k} Q_{m}^{\prime}\left(s^{2}\right)
$$

where $s=S_{2 m}, Q_{m}^{\prime}=J_{m}-Q_{m}$, and $k$ is any odd integer.

Proof. Since $p_{2 m}=p_{m}+q_{m}, q_{2 m}=p_{m}+\left(m-q_{m}\right), r_{2 m}=2 p_{m}$ are solutions of the conditions (3) and (4) for $n=2 m$ whenever $p_{m}, q_{m}, r_{m}$ are solutions of (3) and (4) for $n=m$, it is sufficient to show that $P_{2 m}$ and $Q_{2 m}$ satisfy the condition (2), i.e.

$$
P_{2 m} P_{2 m}^{T}+Q_{2 m} Q_{2 m}^{T}=m I_{2 m}+2 p_{m} J_{2 m} \text {. }
$$

From (**), the left side of (5) equals, (since $P^{T}(s)=P\left(s^{-1}\right)$ ),

$$
\begin{aligned}
\left(P\left(s^{2}\right) P\left(s^{-2}\right)+Q\left(s^{2}\right) Q\left(s^{-2}\right)\right)+\left(P\left(s^{2}\right) P\left(s^{-2}\right)+Q^{\prime}\left(s^{2}\right) Q^{\prime}\left(s^{-2}\right)\right) & \\
& +\left[s^{k} P\left(s^{-2}\right)+s^{-k} P\left(s^{2}\right)\right] J_{m}\left(s^{2}\right), \quad\left[\text { since } Q\left(s^{2}\right)+Q^{\prime}\left(s^{2}\right)=J_{m}\left(s^{2}\right)=J_{m}\left(s^{-2}\right)\right] \\
= & \frac{1}{2} m I+r_{m} \sum_{i=0}^{m-1} s^{2 i}+\frac{1}{2} m I+\left(2 p_{m}-r_{m}\right) \sum_{i=0}^{m-1} s^{2 i}+2 p_{m} \sum_{i=0}^{m-1} s^{2 i+1} \\
= & m I+2 p_{m} J .
\end{aligned}
$$

Let $N_{4 n}$ be a $4 n \times 4 n$ matrix such that

$$
N_{4 n}=\left[\begin{array}{rrrr}
A, & B, & C, & D \\
-B, & A, & -D, & C \\
-C, & D, & A, & -B \\
-D, & -C, & B, & A
\end{array}\right]
$$


where $A, B, C, D$ are $n \times n$ symmetric circulant $(+1,-1)$-matrices. Then a sufficient condition for $N_{4 n}$ being a $W_{4 n}$-matrix is that

$$
N_{4 n} N_{4 n}^{T}=4 n I_{4 n} \text {. }
$$

Let $P, Q, K$, and $G$ be matrices obtained by replacing -1 by 0 in $A, B, C$, and $D$, respectively. Then, corresponding to the conditions (2)-(4), we obtain

$$
P^{2}+Q^{2}+K^{2}+G^{2}=\left(t_{n}-r_{n}\right) I+r_{n} J,
$$

where $t_{n}=p+q+k+g ; p, q, k$, and $g$ are the numbers of 1's in each row of $A, B, C$, and $D$, respectively.

$$
(n-2 p)^{2}+(n-2 q)^{2}+(n-2 k)^{2}+(n-2 g)^{2}=4 n .
$$

Similarly, corresponding to the conditions (5)-(8), we obtain

$$
P^{2}+{Q^{\prime 2}}^{2}+K^{2}+{G^{\prime}}^{2}=\left(t_{n}^{\prime}-r_{n}^{\prime}\right) I+r_{n}^{\prime} J,
$$

where $Q^{\prime}=J-Q, G^{\prime}=J-G$, and $t_{n}^{\prime}=p+q^{\prime}+k+g^{\prime} ; q^{\prime}$ and $g^{\prime}$ are, respectively, the numbers of 1 's in each row of $Q^{\prime}$ and $G^{\prime}$.

$$
\begin{gathered}
(n-2 p)^{2}+\left(n-2 q^{\prime}\right)^{2}+(n-2 k)^{2}+\left(n-2 g^{\prime}\right)^{2}=4 n . \\
t_{n}^{\prime}-r_{n}^{\prime}=n . \\
r_{n}^{\prime}=2(p+k)-r_{n} .
\end{gathered}
$$

THEOREM 2. Let $N_{4 m}$ be a given $W_{4 m}$-matrix with odd $m$ satisfying the conditions $\left(2^{\prime}\right),\left(3^{\prime}\right)$ and $\left(4^{\prime}\right)$. Then $N_{8 m}, a W_{8 m}$-matrix, can be found as follows:

$$
\begin{array}{ll}
P_{2 m}(s)=P\left(s^{2}\right)+s^{m} Q\left(s^{2}\right), & Q_{2 m}(s)=P\left(s^{2}\right)+s^{m} Q^{\prime}\left(s^{2}\right), \\
K_{2 m}(s)=K\left(s^{2}\right)+s^{m} G\left(s^{2}\right), & G_{2 m}(s)=K\left(s^{2}\right)+s^{m} G^{\prime}\left(s^{2}\right) ;
\end{array}
$$

where $s=S_{2 m}, Q^{\prime}=J_{m}-Q$, and $G^{\prime}=J_{m}-G$.

Proof. We know that $P_{2 m}, Q_{2 m}, K_{2 m}$, and $G_{2 m}$ are also symmetric circulant and, as in the proof of Theorem 1, that $p_{2 m}=p+q, q_{2 m}=p+(n-q), k_{2 m}=k+g$, and $g_{2 m}=k+(n-g) ; r_{2 m}=2(p+k)$ are solutions of $\left(3^{\prime}\right)$ and $\left(4^{\prime}\right)$ for $n=2 m$ whenever $p, q, k, g$, and $r_{m}$ are solutions of $\left(3^{\prime}\right)$ and $\left(4^{\prime}\right)$ for $n=m$. Therefore, it is sufficient to prove that the condition $\left(2^{\prime}\right)$ is also satisfied, i.e.

$$
P_{2 m}^{2}+Q_{2 m}^{2}+K_{2 m}^{2}+G_{2 m}^{2}=2 m I+2(p+k) J .
$$

The condition $\left(2^{\prime \prime}\right)$ can be checked easily since the process of proof is exactly similar to that of Theorem 1 .

Let $\left\{u_{i}\right\}$ and $\left\{v_{i}\right\}$ be two finite sequences respectively of

$$
P P^{T}=\sum_{i=0}^{n-1} u_{i} S^{i} \quad \text { and } \quad Q Q^{T}=\sum_{i=0}^{n-1} v_{i} S^{i},
$$

where $P, Q$ are $n \times n$ circulant $(0,1)$-matrices; in this case, we also obtain $w_{n-i}=w_{i}$ for $w=u$ or $v$.

The following Table I, of all constructible $V_{2 n}(1 \leqq n \leqq 16)$ of type $\left(^{*}\right)$ with the restriction $p_{n} \leqq q_{n} \leqq \frac{1}{2} n$, is obtained by matching two finite sequences $\left\{u_{i}\right\}$ and 
$\left\{v_{i}\right\}$, respectively of $P P^{T}$ and $Q Q^{T}$, such that $u_{i}+v_{i}=r_{n}$ for $1 \leqq i \leqq \frac{1}{2} n$. Here, Theorem 1 serves as a tool of classifying these finite sequences.

Note. 1. $s=S_{n}^{k}$, where $k$ is any integer relatively prime to $n$.

2. When $q_{n}=\frac{1}{2} n, Q_{n}(s)$ and $Q_{n}^{\prime}(s)$ produce the same finite sequence.

3. * indicates the class of $P_{n}(s)$ and $Q_{n}(s)$ unobtainable by Theorem 1 .

It should also be noted that for a given $n \times n$ circulant matrix $K(S)$, all matrices $M(i, j)=S^{i} K\left(S^{i}\right)$, for any integers $i$ and $j$ with $(n, j)=1$, produce the same finite sequence corresponding to $M(i, j) M^{T}(i, j)$. Among all $M(i, j)$ regarded as polynomials in $S$, there is a polynomial, say $R$, of least nonnegative degree; we list $R$, as the representative of all matrices $M(i, j)$ producing the same finite sequence, as $R_{n}(s)$ in the Table I.

In Table I, Classes I and II of $n=16$ are respectively derived from the corresponding classes of $n=8$. Although $P_{8}$ and $Q_{8}$ of Class II cannot be derived from $P_{4}$ and $Q_{4}$, they produce $P_{16}$ and $Q_{10}$ of Class II, by Theorem 1 . In this case, $P_{16}$ and $Q_{10}$ are interchangeable since $p=q=6$, and we have

TABLE I

\begin{tabular}{|c|c|c|}
\hline$n$ & $P_{n}(s)$ & $Q_{n}(s)$ \\
\hline 1 & 0 & 0 \\
\hline 2 & 0 & $I$ \\
\hline 4 & $I$ & $I$ \\
\hline $8-\mathrm{I}$ & $I+s$ & $I+s+s^{3}+s^{5}$ \\
\hline II* & $I+s^{2}$ & $I+s+s^{3}+s^{4}$ \\
\hline 10 & $I+s+s^{3}$ & $I+s+s^{4}+s^{6}$ \\
\hline $16-I$ & 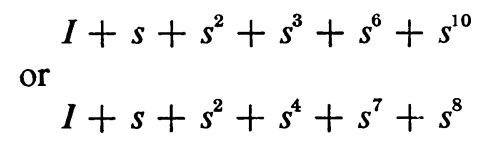 & $\begin{array}{l}I+s+s^{3}+s^{6}+s^{8}+s^{12} \\
\text { or } \quad I+s+s^{4}+s^{6}+s^{8}+s^{11}\end{array}$ \\
\hline II & 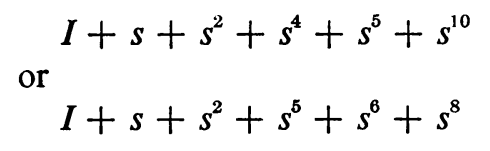 & $\begin{array}{l}\quad l+s+s^{3}+s^{7}+s^{9}+s^{12} \\
\text { or } I+s+s^{4}+s^{7}+s^{9}+s^{11}\end{array}$ \\
\hline III* & $\begin{array}{l}I+s+s^{2}+s^{4}+s^{6}+s^{9} \\
\text { or } \quad I+s^{2}+s^{3}+s^{4}+s^{6}+s^{11} \\
\text { or } \quad \begin{array}{l} \\
I+s+s^{3}+s^{5}+s^{7}+s^{8}\end{array}\end{array}$ & $\begin{array}{l}\text { or } I+s+s^{5}+s^{7}+s^{3}+s^{11} \\
\quad I+s+s^{2}+s^{6}+s^{9}+s^{12} \\
\text { or } \quad I+s+s^{4}+s^{6}+s^{9}+s^{10}\end{array}$ \\
\hline
\end{tabular}




$$
\begin{aligned}
& P(s, k)=P_{8}\left(s^{2}\right)+s^{k} Q_{8}\left(s^{2}\right)=I+s^{4}+s^{k}\left(I+s^{2}+s^{6}+s^{8}\right), \\
& Q(s, k)=P_{8}\left(s^{2}\right)+s^{k} Q_{8}^{\prime}\left(s^{2}\right)=I+s^{4}+s^{k}\left(s^{4}+s^{10}+s^{12}+s^{14}\right) .
\end{aligned}
$$

We obtain

$$
P_{16}(s)=I+s+s^{2}+s^{4}+s^{5}+s^{10}=s Q(s, 5)
$$

or

$$
=I+s+s^{2}+s^{5}+s^{6}+s^{8}=s P(s,-1),
$$

since these two polynomials are of distinct type (in the sense of [5]) and of least positive degree in $s=S$ producing the same finite sequence among all $P(s, k)$ and $Q(s, k)$ for this case.

When $n=20$, we obtain two subclasses of matrices $P$ and $Q$ by Theorem 1 . We have the following cases:

Subclass-1:

$$
P(s, k)=P_{10}\left(s^{2}\right)+s^{-k} Q_{10}\left(s^{2}\right)=I+s^{2}+s^{6}+s^{-k}\left(I+s^{2}+s^{8}+s^{12}\right)
$$

and

$$
\begin{aligned}
Q(s, k) & =P_{10}\left(s^{2}\right)+s^{-k} Q_{10}^{\prime}\left(s^{2}\right) \\
& =I+s^{2}+s^{6}+s^{-k}\left(s^{4}+s^{6}+s^{10}+s^{14}+s^{16}+s^{18}\right)
\end{aligned}
$$

Subclass-2:

$$
\begin{aligned}
P(s, k) & =P_{10}\left(s^{2}\right)+s^{-k} Q_{10}\left(s^{-2}\right) \\
& =I+s^{2}+s^{6}+s^{-k}\left(I+s^{-2}+s^{-8}+s^{-12}\right)
\end{aligned}
$$

and

$$
\begin{aligned}
Q(s, k) & =P_{10}\left(s^{2}\right)+s^{-k} Q_{10}^{\prime}\left(s^{2}\right) \\
& =I+s^{2}+s^{6}+s^{-k}\left(s^{4}+s^{6}+s^{10}+s^{14}+s^{16}+s^{18}\right) ;
\end{aligned}
$$

Each one of the subclasses produces five distinct designs corresponding to $k=1,3$, 5,7 , and 9. For example, the finite sequence $\left\{u_{2 i+1}\right\}$ of odd components (since the even components $u_{2 i}=r=2$ for all $i$, it is sufficient to consider only odd components of $\left.\left\{u_{i}\right\}\right)$ corresponding to $P(S, k)$ are: $\left(u_{1}, u_{3}, u_{5}, u_{7}, u_{9}\right)=(4,1,3,2,2),(2,4,2,2,2)$, $(2,3,3,2,2),(3,1,3,3,2)$, and $(2,3,1,3,3)$ for Subclass-1 respectively of $k=1,3$, 5, 7, and 9; and (2, 2, 3, 2, 3), (1, 3, 3, 2, 3), (2, 2, 2, 4, 2), (3, 1, 3, 3, 2), (2, 4, 1, 2, 3) for Subclass-2.

The following Table II is obtained by taking $s=S^{k}$ with $k$, an integer relatively prime to $n=20$ for $P_{20}=P(s, 9)$ of Subclass-2, i.e. $P_{20}\left(S^{k}\right)=I+S^{2 k}+S^{3 k}+S^{6 k}+$ $S^{9 k}+S^{11 k}+S^{19 k}$.

Starting from $P=Q=I$ for $n=4$, and repeating applications of Theorem 1, we obtain, for example, the following $P_{n}, Q_{n}$ for $n=32$ and 64:

$$
P_{32}=\sum_{\alpha} s^{\alpha}, \quad \text { where } \alpha \in\{0,1,2,3,4,8,9,13,14,16,17,23\}
$$

and

$Q_{32}=\sum_{\beta} s^{\beta}, \quad$ where $\beta \in\{0,2,4,5,7,8,11,14,15,16,19,21,25,27,29,31\}$;

$$
P_{64}=\sum_{\alpha} s^{\alpha}, \quad Q_{64}=\sum_{\beta} s^{\beta},
$$


TABLE II

\begin{tabular}{|c|c|c|c|c|}
\hline \multirow{2}{*}{$\frac{k}{1}$} & \multicolumn{3}{|c|}{$(+1,-1)$-matrix $A$ corresponding to $P_{20}$} & \multirow{2}{*}{$\frac{\left\{u_{2 \imath+1}\right\}}{2,4,1,2,3}$} \\
\hline & +-++--+--+ & -+--- & ----+ & \\
\hline 3 & $+----\quad++-+$ & ---+- & --++- & $2,2,1,3,4$ \\
\hline 7 & $++++-\quad-----$ & ---++ & --+-- & $4,3,1,2,2$ \\
\hline 7 & ++-----+-- & -+--+ & ---++ & $3,2,1,4,2$ \\
\hline
\end{tabular}

where $\alpha \in\{0,1,2,4,5,6,8,9,11,15,16,17,18,23,26,28,29,31,32,33,34,39$, $43,46,51,55,59,63\}$ and $\beta \in\{0,2,3,4,6,7,8,13,16,18,19,21,25,26,27,28,32$, $34,35,37,41,45,46,47,49,53,57,61\}$.

It should be noted that Theorem 3 of Williamson [4] produces Williamson type matrices of the same order, but of different construction, as given by Theorem 2 of this paper. When $n=29$, we obtain a $W_{4 n}$-matrix (see [7]) with submatrices

$$
P_{29}=\sum_{\alpha} t_{\alpha}, \quad Q_{29}=\sum_{\beta} t_{\beta}, \quad K_{29}=\sum_{\gamma} t_{\gamma}, \quad G_{29}=\sum_{\delta} t_{\delta},
$$

where $t_{k}=S^{k}+S^{29-k} ; \alpha \in\{2,3,5,6,8,12\}, \beta \in\{4,7,9,10,11\}, \gamma \in\{3,4,5,8$, $9,11,13,14\}$, and $\delta \in\{1,3,4,5,8,9,11\}$. By applying Theorem 2 , we obtain $W_{8 n^{-}}$ matrix with submatrices

$$
P_{58}=\sum_{\alpha} t_{\alpha}, \quad Q_{58}=\sum_{\beta} t_{\beta}, \quad K_{58}=\sum_{\gamma} t_{\gamma} \quad \text { and } \quad G_{58}=\sum_{\delta} t_{\delta},
$$

where $t_{k}=s^{k}+s^{58-k}$ for $k \neq 29$ and $t_{29}=s^{29}$; and $\alpha \in\{4,6,7,9,10,11,12,15$, $16,21,24\}, \beta \in\{1,3,4,5,6,10,12,13,16,17,19,23,24,25,27,29\}, \gamma \in\{6,7,8$ $10,11,13,16,18,19,21,22,23,26,27,28\}$, and $\delta \in\{1,3,5,6,8,9,10,15,16,17$, $18,22,25,26,28,29\}$.

State University College

Oneonta, New York 13820

1. L. D. Baumert \& Marshall Hall, JR., "Hadamard matrices of the Williamson type," Math. Comp., v. 19, 1965, pp. 442-447. MR 31 \#3344. 37 \#80.

2. Marshall Hall, JR., Combinatorial Theory, Blaisdell, Waltham, Mass., 1967. MR

3. H. J. Ryser, Combinatorial Mathematics, Carus Math. Monographs, no. 14, Math. Assoc. Amer., distributed by Wiley, New York, 1963. MR 27 \#51.

4. J. Williamson, "Hadamard's determinant theorem and the sum of four squares," Duke Math. J., v. 11, 1944, pp. 65-81. MR 5, 169.

5. C. H. YANG, "On designs of maximal $(+1,-1)$-matrices of order $n \equiv 2(\bmod 4)$," Math. Comp., v. 22, 1968, pp. 174-180. MR 37 \#1069.

6. C. H. YANG, "On designs of maximal $(+1,-1)$-matrices of order $n \equiv 2(\bmod 4)$. II," Math. Comp., v. 23, 1969, pp. 201-205. MR 39 \#1105.

7. L. D. BAUMERT, "Hadamard matrices of orders 116 and 232," Bull. Amer. Math. Soc., v. 72, 1966, p. 237. MR 32 \#4026. 